\title{
Design and Development of a Novel 3-D Cubic Antenna for Wireless Sensor Networks (WSNs) and RFID Applications
}

\author{
Catherine M. Kruesi, Member, IEEE, Rushi J. Vyas, Student Member, IEEE, and \\ Manos M. Tentzeris, Senior Member, IEEE
}

\begin{abstract}
A novel miniaturized 3-D cubic antenna for use in a wireless sensor network (WSN) and RFIDs for environmental sensing is introduced. The antenna produces a truly omnidirectional pattern in both $E$-plane and $H$-plane, which allows for non-intermittent communication that is orientation independent. The frequency of operation lies in the UHF RFID band, 902 MHz-928 MHz (centered at $915 \mathrm{MHz}$ ). The ultra-compact cubic antenna has dimensions of $3 \mathrm{~cm} \times 3 \mathrm{~cm} \times 3 \mathrm{~cm}\left(27 \mathrm{~cm}^{3}\right)$, which features a length dimension of $\lambda / 11$. The cubic shape of the antenna allows for "smart" packaging, as sensor equipment may be easily integrated inside the cube's hollow (or Styrofoam-filled) interior. The prototype fabrication was performed on six (planar) sides on liquid crystal polymer (LCP) substrate, and then folded into the cubic structure. The geometry of the design is inspired by the RFID inductively coupled meander line structures, which are folded around the sides of the cube. Due to the large number of freedom degrees, this antenna concept may be easily reconfigured for many values of impedances and design parameters. Experimental data verify the simulation results.
\end{abstract}

Index Terms-Dipole antennas, flexible, folded antennas, liquid crystal polymer (LCP), omnidirectional antennas, smart packaging, UHF antennas, wireless sensor networks (WSNs), 3-D antennas.

\section{INTRODUCTION}

$\mathbf{T}$ HE use of wireless sensor networks (WSNs), which consist of spatially distributed autonomous nodes, that communicate with each other, and a base station through multi-hopping, has recently grown as a technology. Although originally used for defense purposes, WSNs can be used for civilian purposes such as real-time monitoring and tracking of environmental conditions. The motivation for this paper is to use WSNs for the tracking of weather, specifically tornadoes. The design of the presented cubic antenna aimed in combining an omnidirectional radiation pattern, low weight, capability of having sensors

Manuscript received December 10, 2008; revised March 06, 2009. First published August 07, 2009; current version published October 07, 2009. This work was supported by the Georgia Institute of Technology.

C. M. Kruesi was with Georgia Institute of Technology, Atlanta, GA 30223. She is now with The Aerospace Corporation, Chantilly, VA 20151 USA (e-mail: catherine.m.kruesi@aero.org).

R. J. Vyas and M. M. Tentzeris are with the Electrical and Computer Engineering Department, Georgia Institute of Technology, Atlanta, GA 30332 USA (e-mail: rushi.vyas@gatech.edu; etentze@ece.gatech.edu).

Color versions of one or more of the figures in this paper are available online at http://ieeexplore.ieee.org.

Digital Object Identifier 10.1109/TAP.2009.2028672 packaged within the antenna structures, and efficient matching at $915 \mathrm{MHz}$ (high power efficiency). The reason for using the UHF RFID band is that this antenna can be exploited for use as an RFID antenna for the tracking of position and velocity within a tornado. As will be demonstrated, a wide range of impedances can be realized by modifying the many design parameters inherent with using six sides as a basis for the antenna geometry. The cubic antenna design has the potential to be the first truly 3-D ultra-low-cost solution for the realization of truly ubiquitous wireless sensing applications, especially when fabricated on flexible, organic liquid crystal polymer (LCP) or paper.

The use of a cubic structure with integrated planar antenna structures has been studied for MIMO applications ([1]-[4]). These cubes have many antennas mounted on them with each one fed by a different port. In this case, isolation between ports is desirable. However, the use of a cubic structure with a single input where the planar antenna structures are intentionally coupled to improve radiation has not been previously published. The use of all six sides for one radiating structure allows for homogeneous radiation into $4 \pi$ steradians in space.

In this paper, the design of a miniaturized, cubic antenna for use as the radiation part of an enclosed sensor in a wireless sensor node is proposed. The concept is based on meander line dipole antenna configurations ([5]-[7]), folded into a cube structure. Due to the optimized positioning of the arms of the dipole and using inductive coupling matching techniques, a matched, isotropic radiator is achieved.

\section{Cubic Antenna Design}

\section{A. Antenna Design}

Dipole meander line structures have been used for the purpose of size reduction in UHF antennas ([5]-[7], [9], and [10]). The arms of the dipole are commonly folded in order to reduce the height/side dimension of the dipole, while sacrificing bandwidth and efficiency. As discussed in [5], Genetic Algorithms may be used to guide the shape of the meander line. Fig. 1 shows the geometry of the meander line for one dipole arm. The design of Fig. 1(b) is chosen for enhanced bandwidth performance. Due to the intrinsic operation of the dipole, with current at the ends of the arms going to zero, it is desirable to have less meandering closer to the feed element where the highest concentration of current is located. The horizontal segments of the meander lines will not contribute to the radiated power of the antenna because the line currents will cancel in phase. By allowing the meanders to be concentrated at the ends of the dipole arms in order to 


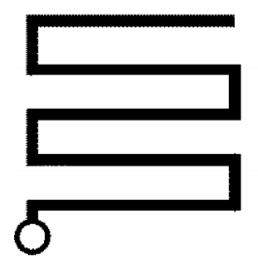

(a)

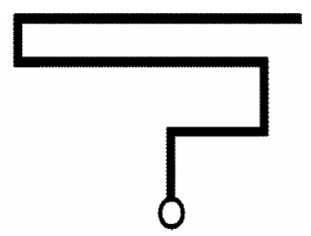

(b)
Fig. 1. Meander line dipole arm structures. (a) Uniform meander line and (b) nonuniform meander line.

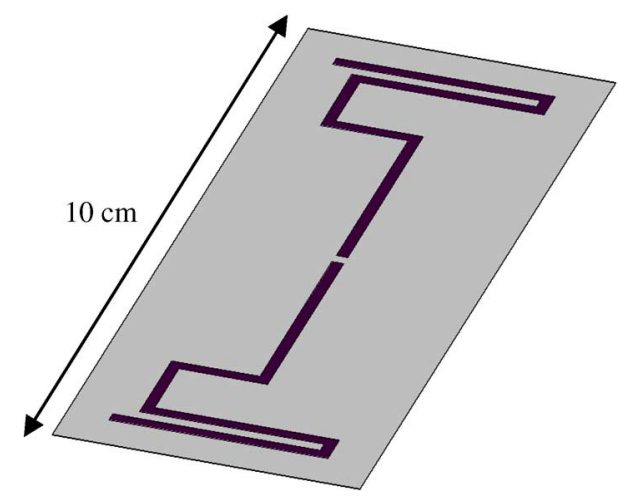

Fig. 2. Planar meander line structure.

minimize the horizontal lengths, a larger impedance bandwidth and higher efficiency may be realized [5]. The goal of using a cubic structure in conjunction with the meander line dipole would be to investigate whether further height reduction could be accomplished while preserving or improving matching and radiation characteristics.

The substrate for the antenna was chosen to be LCP due to its excellent electrical and mechanical performance [8]. With a low dielectric constant $\left(\varepsilon_{\mathrm{r}}=3\right)$ and loss tangent $(\tan \delta=0.002)$ which allow for higher efficiencies and mechanical flexibility, a cubic structure can be easily fabricated through bending six sides. LCP proved to be a very good material for this application.

Before analyzing the cubic structure, it will be beneficial to first look at a planar meander line antenna. The proposed planar antenna is shown in Fig. 2. The meandering of the dipole arms will allow for minimization in the length dimension. The $\mathrm{S}_{11}$ data for this antenna is shown in Fig. 3. The antenna is resonant at $900 \mathrm{MHz}$, which corresponds to a $\lambda / 2$ dipole length of $16.7 \mathrm{~cm}$. The radiation pattern at resonance is the typical donut shaped pattern with a directivity of $2.01 \mathrm{~dB}$. The length of the meander line dipole is $8.9 \mathrm{~cm}$, which is a $53 \%$ reduction in length.

The planar antenna has some advantages in size miniaturization, but the radiation pattern does not cover the full $360^{\circ}$ we desire for our application. We want to investigate how this antenna structure will behave when folded into a cube.

Using Ansoft's HFSS for simulations, it was found that when the meander line antenna was folded over the sides of the cubic structure, the impedance became capacitive due to the coupling between the meander lines on either side of the cube. The dipole arms on either side of the antenna will have currents that are $180^{\circ}$ out of phase with each other and will act as a capacitor.

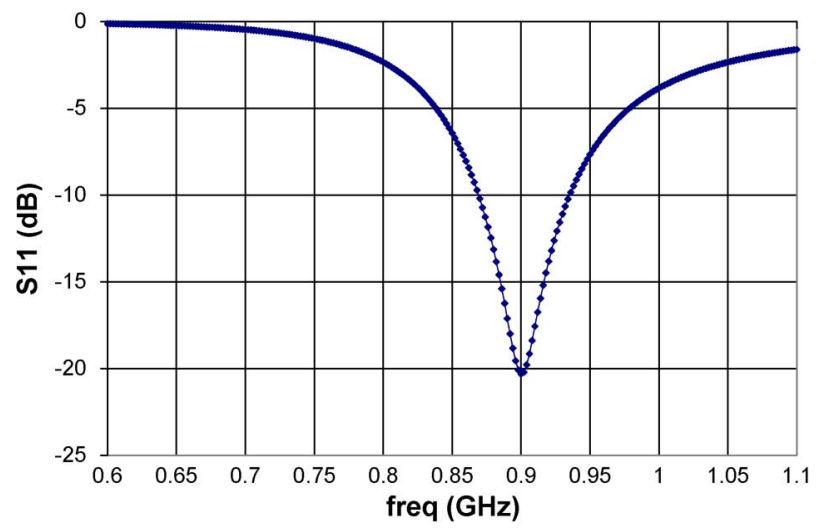

Fig. 3. $\mathrm{S}_{11}$ for planar meander line antenna shown in Fig. 2.

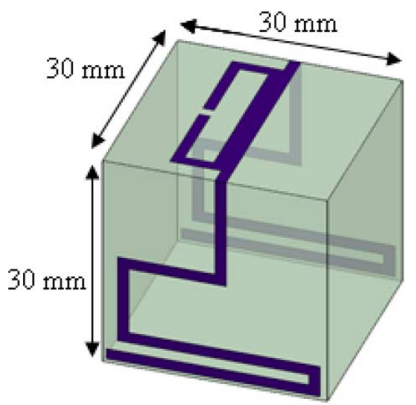

Fig. 4. Folded meander line antenna structure.

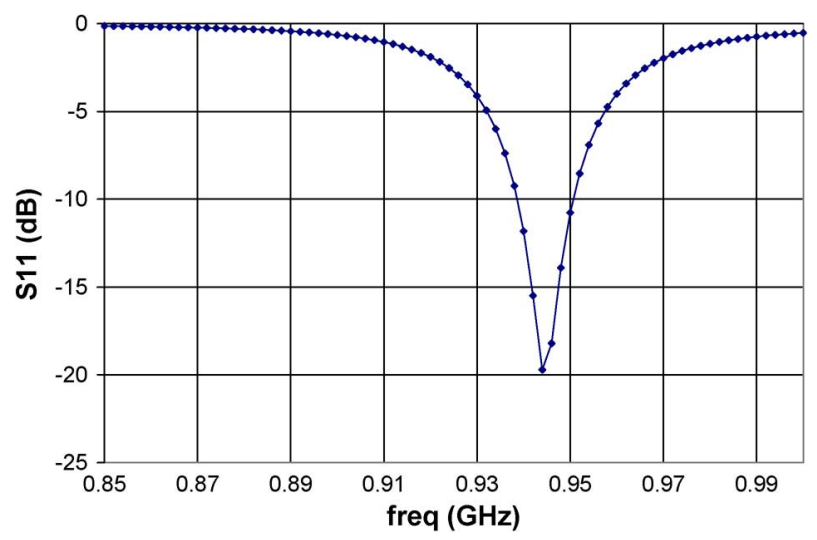

Fig. 5. $\mathrm{S}_{11}$ of antenna shown in Fig. 4.

The folded antenna is shown in Fig. 4. An inductively coupled loop was added as the feeding structure similar to [9] in order to cancel the capacitance. However, through optimization, a better match (to $50 \Omega$ ) was found by using a T-match configuration, discussed in [10]. The $\mathrm{S}_{11}$ characteristic for the folded dipole is shown in Fig. 5.

The inductive couple and the capacitance cancel at the resonant frequency. In addition, the effect of folding also decreases the overall bandwidth to about $1.3 \%$. The radiation pattern still has a null in the pattern, so we will want to improve this by adding a parasitic element.

The ideal resonant frequency for this antenna is $915 \mathrm{MHz}$. This antenna is resonant at a higher frequency, but this shift may be accounted for by introducing a symmetric structure on the other three sides of the cube. 


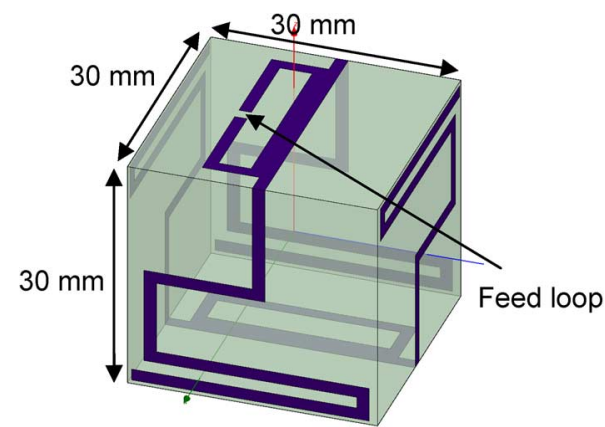

Fig. 6. Optimized cubic antenna.

The optimized 3-D antenna is shown in Fig. 6. The structures on the top and bottom of the antenna are identical, except that the bottom element has no excitation slot and is purely parasitic. As will be shown, the parasitic element couples to the driven element and creates an increase in radiation efficiency, and will allow for miniaturization. The structure on the bottom of the antenna is rotated by $90^{\circ}$ for full usage of the six sides and radiation into all spatial directions. The symmetry in the structure is important as will be demonstrated in Section III of this paper. When symmetry is broken in the structure, multiple resonances will occur which, in this application, is undesirable due to the effective perturbation of the omni pattern. Each side of the cube is $30 \mathrm{~mm}(\sim \lambda / 11)$, and the "shell" (cubic sides) of LCP material is 2 mil thick. Section III will discuss simulation and measurement data detailing the response of the antenna as well as a discussion of how optimization was conducted.

\section{B. RFID Applications}

The motivation for using the cubic antenna in an RFID-type application is using RFID-enabled sensors, such as gas sensors that require a large gas-interaction area. Also, this cube could be used as a pallet/container 3-D conformal antenna. If this antenna were to be used with an RFID IC chip, we would need to reconfigure the antenna in a way that it would be conjugately matched to the complex impedance. The beauty of the cubic antenna is that the parameters of the antenna, specifically the size and position of the inductive couple and meander line can be manipulated to match to any desired impedance [11]. Changing the size of the inductive loop will change the associated reactance to conjugately match to the desired impedance. By changing the length of the meander line, the resonant frequency will also shift, as expected of a dipole antenna. By making the total length of the meandering arms longer, the frequency will shift lower, and making the arms shorter will cause an increase in the resonant frequency. Therefore, when using the cube antenna for RFID applications, we may vary the length of the meander line and the size of the inductive couple to match any complex impedance, which is ideal for most RFID IC chips.

\section{Cubic Antenna Analysis}

\section{A. Optimization of Folding Effect}

To fully understand exactly which parameters affected the matching as well as the radiation pattern, it was beneficial to analyze the antenna with even symmetry and odd symmetry. Even

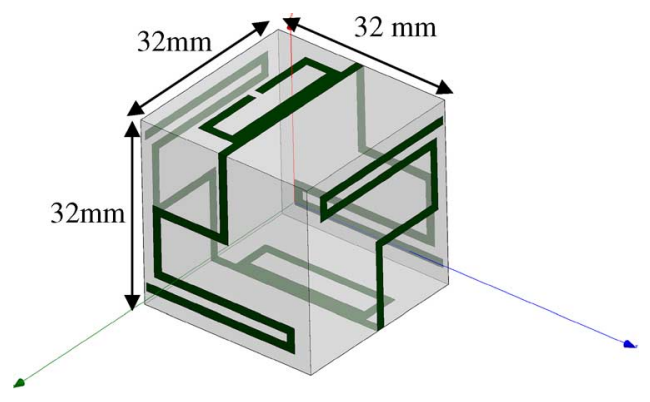

Fig. 7. Oddly symmetric cube.

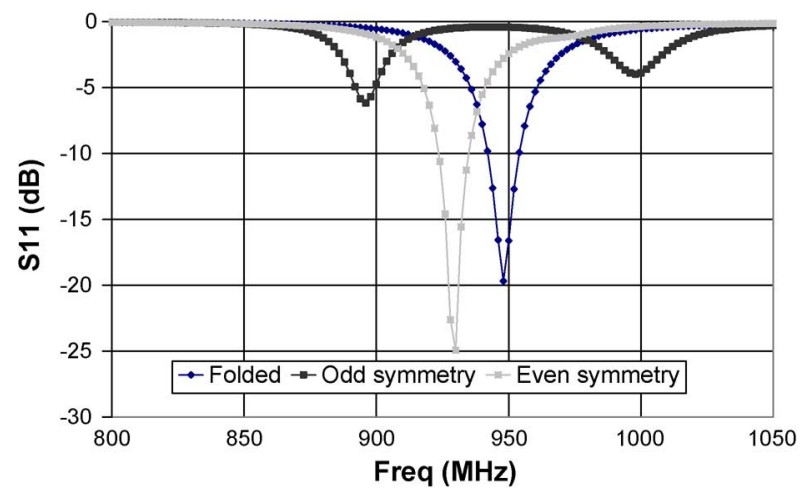

Fig. 8. S-parameter simulation results of folded, odd-symmetric, and evensymmetric versions of the cubic antenna.

symmetry implies that the nonuniform meander line structure has even symmetry with respect to the $z$-axis, as depicted in Fig. 6. Odd symmetry would occur if the meander line is oddly symmetric with respect to the $z$-axis, or that the meander line on the front ( $y z$ plane) is rotated by $180^{\circ}$ to form the back meander line of the $y z$ plane (and then joined along the top of the structure). This geometry can be referenced in Fig. 7, with the meander line length and loop size being identical to that of Fig. 6 . However, due to the geometry of the meander line, it was necessary to expand the cube size to $32 \mathrm{~mm}$ per side.

The graph in Fig. 8 shows the S-parameters, simulated in Ansoft's HFSS, of the configurations of the meander line structure discussed in the previous paragraph along with the folded dipole presented in Section II.

The oddly symmetric configuration produces two resonances. Based on the results of multiple simulations with a variety of meander line lengths and cubic size, this same pattern occurs whenever the nonuniform meander line has odd symmetry. This is due to the current distribution of the antennas. In odd mode, the corners of the antenna create an equivalent RLC circuit. The active element is the driven element where the feed is located (top structure), whereas the passive element is the bottom structure which has no excitation. The two resonating structures in odd-mode cubic configurations behave differently at the two resonances. At the first resonance, the Electric field in the active structure will peak before the passive structure, whereas, at the second resonance, the passive structure will couple to the feed and peak before the active one (Fig. 9). This effect will also cause a $90^{\circ}$ shift in the radiation pattern as shown in Fig. 9. The dual resonance occurs because the cube is acting as four dipoles. The first resonance occurs when the active element is radiating 


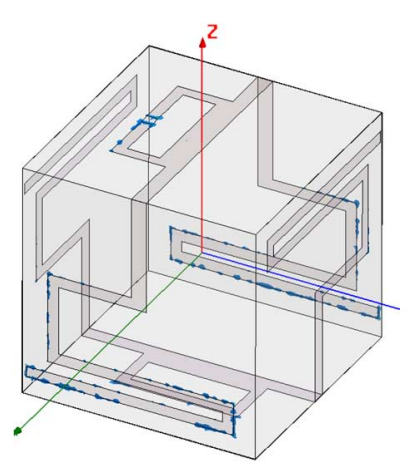

(a)

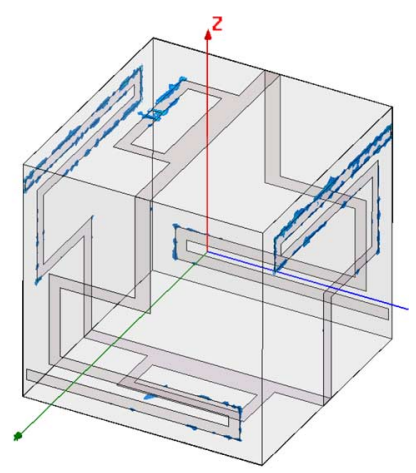

(b)
Fig. 9. E-field in the substrate of the cube for odd mode configurations at (a) $890 \mathrm{MHz}$ and (b) $988 \mathrm{MHz}$.

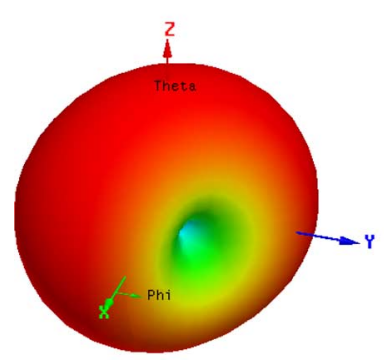

(a)

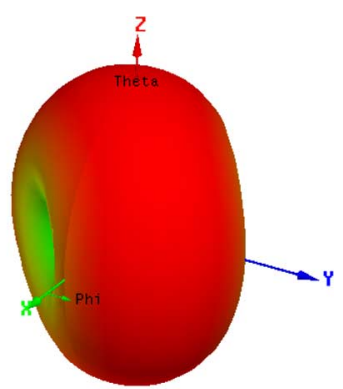

(b)
Fig. 10. Radiation pattern for odd-mode cubic antenna at (a) $890 \mathrm{MHz}$ and (b) $988 \mathrm{MHz}$.

and couples to the passive. The higher order mode is the resonance of the feed coupling to the passive element directly. The excitation in the first frequency excites the vertical direction in the front and back of the antenna, whereas the higher frequency will couple to the horizontal elements and excite a higher order mode. If the excitation then is placed on the bottom element, the radiation pattern will switch and the higher order mode pattern will be that shown in Fig. 10(a) and the lower order mode pattern will be that shown in Fig. 10(b). The directivity of both patterns is $2.2 \mathrm{~dB}$ (simulated in HFSS).

Since the goal of this antenna is resonance at a single frequency in the North American ISM band, the even symmetry is preferred. The even symmetry causes an E-field in the substrate as shown in Fig. 11. Here, all four dipole structures will be excited at the resonant frequency. The effect of the parasitic element can be observed from Fig. 6. There is a resonance shift to a lower frequency ( $\sim 20 \mathrm{MHz}$ ) when using parasitic coupling of the bottom structure to the top, due to an increase in radiation impedance. Since the odd-mode structure has complicated coupling effects, this same shift cannot be seen in the odd mode antenna without the bottom element. However, only one resonance is present without the parasitic coupler, as expected. In the even mode antenna, the frequency shift is advantageous for the reduction in size of the overall cube. Through these optimization simulations, the cube depicted in Fig. 6 is chosen for fabrication and measurement. The resonance of this antenna is at $930 \mathrm{MHz}$, but as will be shown, this is optimal to account for the frequency shift that occurs with fabrication due to the non-ideal
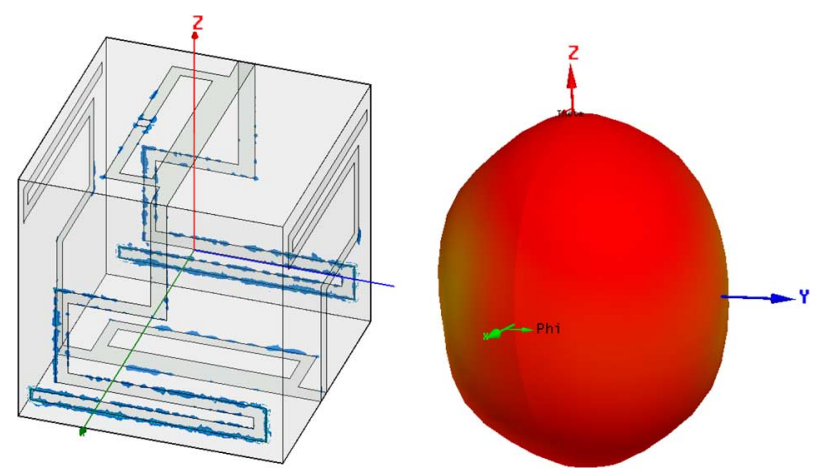

Fig. 11. E-field (a) and 3-D radiation pattern (b) of cubic antenna shown in Fig. 6.

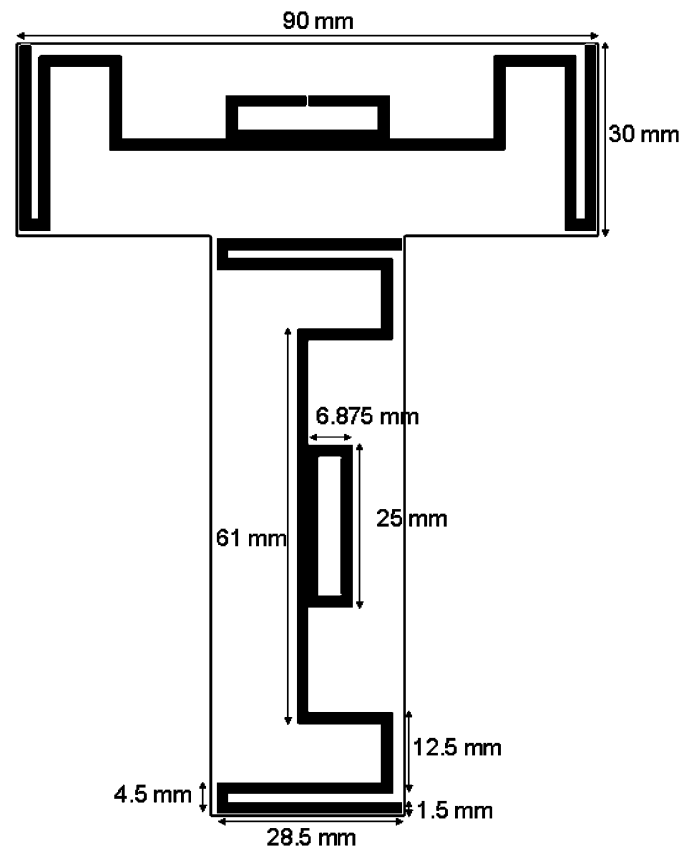

Fig. 12. Planar antenna before folding with dimensions.

components that will be used. These components will introduce loss and cause the antenna to peak at a lower frequency. At resonance, the radiation pattern is shown in Fig. 11.

As shown, the radiation pattern is almost truly isotropic with a slight E-plane tendency. The axial ratio is primarily above 5 in most directions, except where the cube edges occur and the axial ratio drops below 2, indicating that the polarization is largely linear except on the cube edges where the adjacent meander lines create an elliptical polarization.

\section{B. Fabrication and Measurements}

The antenna in Fig. 6 is fabricated by first performing photolithography on planar LCP and then folding the T into a cube. The planar form of the antenna is shown in Fig. 12, and the cubic form in Fig. 13. For measurements, some versions of the cube were fabricated around a cube of Styrofoam. The advantage of using Styrofoam is that its dielectric constant is very close to air, which does not cause a significant variance to performance, and it enhances the mechanical stability. Since the application 


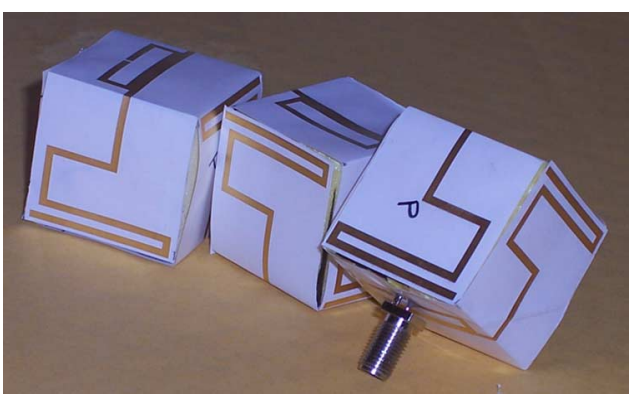

Fig. 13. Manufactured cubic antenna.

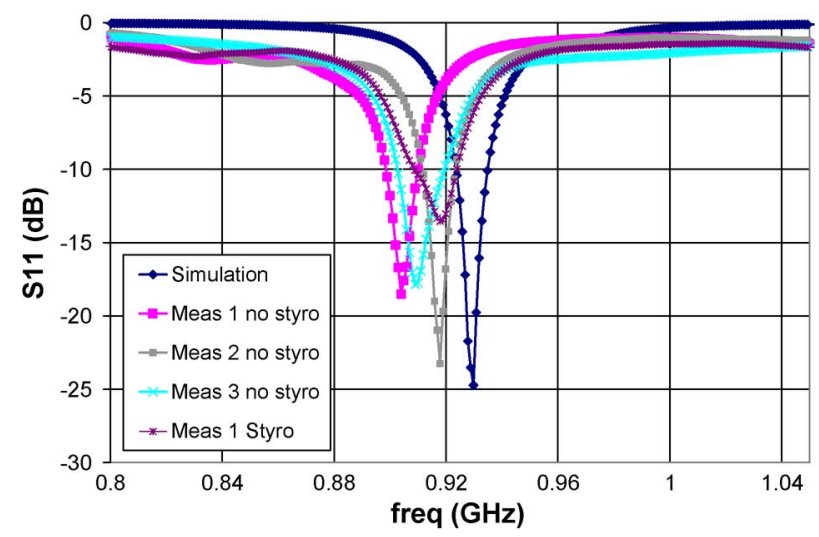

Fig. 14. S-parameter measurements, measurement compared to simulation data.

is in a turbulent environment, the Styrofoam assembly is preferred. The measured along with the simulation S-parameters are shown in Fig. 14.

The antenna is measured with an SMA that has been attached by drilling holes into the copper at the feed, inserting the SMA pins and soldering the feed to input of the antenna as can be seen in Fig. 13. Although the SMA is an unbalanced feed, whereas the dipole is a balanced feed, it does not significantly affect the measurements. The measurement facility uses a magnetic core that allows for only a minor deterioration in radiation pattern, despite the unbalanced feed.

As shown in Fig. 14, there is a frequency shift from the resonance at $930 \mathrm{MHz}$. These frequency shifts are different for each cube that was fabricated. However, each cube is resonant around the desired $915 \mathrm{MHz}$. The reason for the shift in frequency is largely due to the inaccuracies inherent in fabrication. The edges of the cube were taped together and the edges not exactly at 90 degree angles. The crudeness of fabrication accounts for the frequency shifts and as is evident in the measurements, the Styrofoam adds some additional discrepancies compared to the simulations. The frequency shifting actually improves our results by making the antenna resonant at the desired frequency.

Radiation pattern measurements were conducted at the Satimo facility in Kennesaw, GA. The radiation patterns in the phi $=90^{\circ}$, phi $=0^{\circ}$ are shown in Fig. 15 and Fig. 16. The full 360 measured radiation pattern (E-total) is shown in Fig. 17 for the cubic antenna fabricated with Styrofoam at $915 \mathrm{MHz}$. The efficiency of the antenna is $75 \%$ at $915 \mathrm{MHz}$ and the maximum gain is $0.53 \mathrm{dBi}$. The lower efficiency is due to the unbalanced

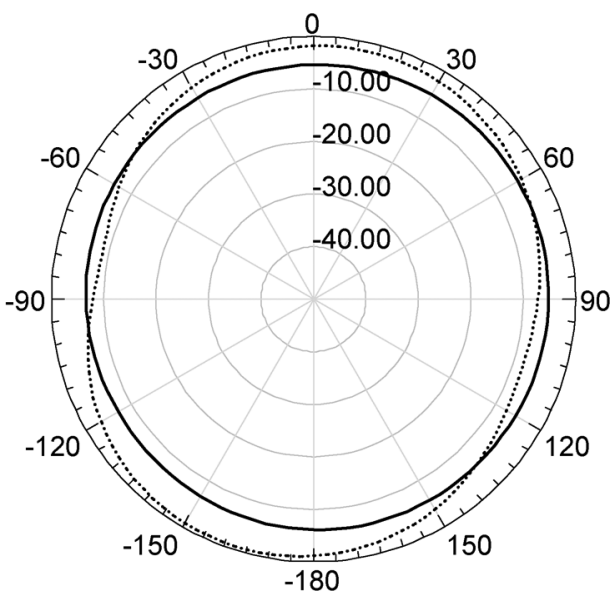

Fig. 15. E-phi (solid) and E-theta (dashed) in $\mathrm{dB}, \mathrm{phi}=90^{\circ}$ for cubic antenna fabricated with Styrofoam.

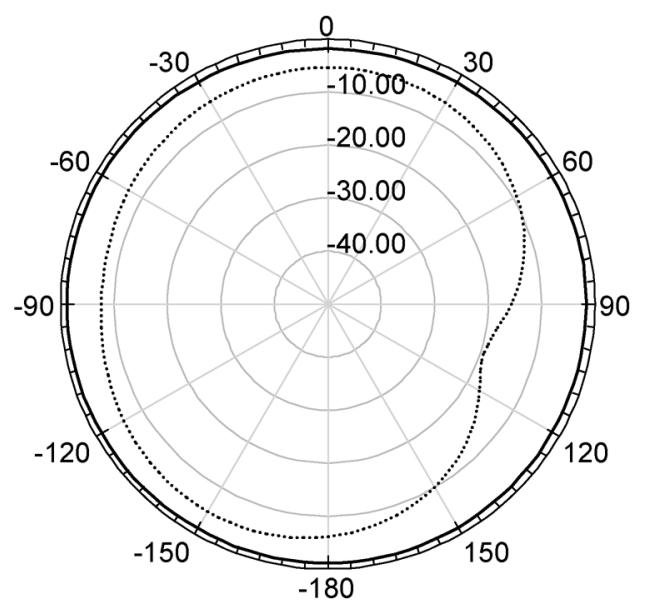

Fig. 16. E-phi (solid) and E-theta (dashed) in $\mathrm{dB}, \mathrm{phi}=0^{\circ}$ for cubic antenna fabricated with Styrofoam.

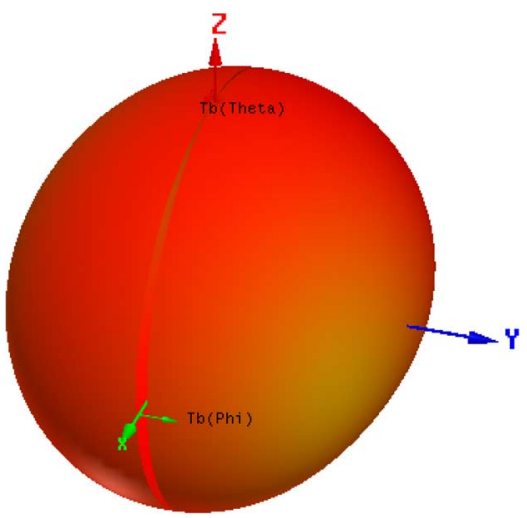

Fig. 17. Radiation pattern for the cube in Fig. 6.

feed during measurements. If possible the antenna should be measured with a balun in order to determine correct efficiency.

Likewise, the radiation pattern measurements for the cubic antenna fabricated without Styrofoam are almost identical to the radiation pattern plots of the antenna with Styrofoam. The efficiency of this antenna is $72 \%$ and the maximum gain is $0.5 \mathrm{dBi}$. 
The slight tilt in the radiation patterns in Fig. 16 is due to the tilt of the antenna on the SMA connector during fabrication.

As can be seen, the radiation pattern measurements are approximately the same for the antennas fabricated with and without Styrofoam. This means that the antenna may be fabricated on Styrofoam for enhanced mechanical stability. Also, it is clear that these antennas may be mass produced and that there will be good agreement between samples. The cross-polarization was checked for both antennas and was about $15 \mathrm{~dB}$, at minimum, below the co-polarization in any given direction. As can be shown in Fig. 15-Fig. 17, the radiation pattern of the cubic antenna is nearly isotropic. These results show that communication between nodes will be possible regardless of the orientation of the cube, making them ideal for use in a WSN.

\section{CONCLUSION}

The design, fabrication, and measurement of a cubic antenna with a nearly isotropic radiation pattern has been achieved. The antenna is matched to $50 \Omega$ in the desired RFID band, and can be reconfigured to match almost any impedance. The cube can also be used for system-in-package technology, whereby the antenna provides housing for sensor electronics, providing the optimum solution for a WSN node. Using this technology, low-cost weather tracking may be realized, especially in turbulent scenarios, such as tornadoes.

\section{ACKNOWLEDGMENT}

The authors would like to thank K. Rutkowski from Satimo in Kennesaw, GA for their support with radiation pattern measurements.

\section{REFERENCES}

[1] C. Y. Chiu and R. Murch, "Experimental results for a MIMO cube," in Proc. IEEE Antennas Propag. Soc. Int. Symp., Albuquerque, NM, 2006, pp. 2533-2536.

[2] C. Y. Chiu and R. Murch, "Design of a 24-port MIMO cube," in Proc. IEEE Antennas Propag. Soc. Int. Symp., Honolulu, HI, 2007, pp. 2397-2400.

[3] A. Nemeth, L. Sziics, and L. Nagy, "MIMO cube formed of slot dipoles," in Proc. IST Mobile and Wireless Commun. Summit, July 2007, pp. 1-5.

[4] B. N. Getu and R. Janaswamy, "The MIMO cube-A compact MIMO antenna," IEEE Trans. Wireless Commun., vol. 4, no. 3, pp. 1135-1141, May 2005.

[5] G. Marrocco, "Gain-optimized self-resonant meander line antennas for RFID applications," IEEE Antennas Wireless Propag. Lett., vol. 2, no. 1, pp. 302-305, Jan. 2003.

[6] K. V. S. Rao, P. V. Nikitin, and S. F. Lam, "Antenna design for UHF RFID tags: A review and a practical application," IEEE Trans. Antennas Propag., vol. 53, no. 12, pp. 3870-3876, Dec. 2005.

[7] L. Yang, S. Basat, and M. M. Tentzeris, "Design and development of novel inductively coupled RFID antennas," in Proc. IEEE Antennas Propag. Soc. Int. Symp., Albuquerque, NM, Jul. 2006, pp. 1035-1038.

[8] D. Thompson, "Characterization and design of liquid crystal polymer (LCP) based multilayer RF components and packages," Ph.D. dissertation, Dept. Elect. Comput. Eng., Georgia Inst. of Technol., Atlanta, GA, 2006.

[9] H. W. Son and C. S. Pyo, "Design of RFID tag antennas using an inductively coupled feed," Electron. Lett., vol. 41, no. 18, pp. 994-996, Sep. 2005.

[10] G. Marrocco, "The Art of UHF RFID antenna design: Impedancematching and size-reduction techniques," IEEE Antennas Propag. Mag., vol. 50, no. 1, pp. 66-79, Feb. 2008.
[11] C. Kruesi and M. M. Tentzeris, “'Magic cube' antenna configurations for ultra compact RFID and wireless sensor nodes," in Proc. IEEE Antennas Propag. Soc. Int. Symp., San Diego, CA, 2008, pp. 1-4.

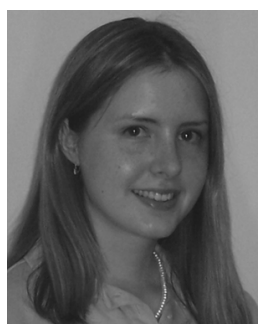

Catherine M. Kruesi (M'04) was born in Phoenixville, PA. She received the B.S. degree (summa cum laude) in electrical engineering from Bucknell University, Lewisburg, PA, in 2007 and the M.S. degree in electrical and computer engineering from the Georgia Institute of Technology (Georgia Tech), Atlanta, in 2008.

At Georgia Tech, she held the Georgia Tech Institute Fellowship. She was a member of the Athena Laboratory in the Georgia Electronic Design Center. Her research interests include small antennas, biomedical applications of electromagnetics, and RFID and backscattering technologies. She is currently with The Aerospace Corporation, Chantilly, VA, where she is a Member of the Technical Staff in the Digital Electronics and Electromagnetics Department.

Ms. Kruesi is a member of Eta Kappa Nu and Tau Beta Pi.

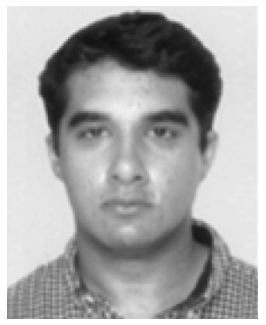

Rushi J. Vyas (S'08) received the B.S. degree in electrical engineering from the Georgia Institute of Technology, Atlanta, in 2005 with a background in $\mathrm{RF}$, analog circuit design, and embedded systems. He is currently pursuing the Ph.D. degree in electrical and computer engineering at the Georgia Institute of Technology.

He is currently a Graduate Research Assistant with the Georgia Electronic Design Center, Atlanta. His works so far have been in areas of antenna design in the millimeter-wave and UHF range, power amplifier design and characterization, and its integration primarily on eco-friendly organic substrates using inkjet printing techniques for automotive radar and RFID applications. His current research involves combining wireless, embedded systems and power electronics techniques in the design and prototyping of enhanced range, power scavenging tags for RFID and remote sensing applications.

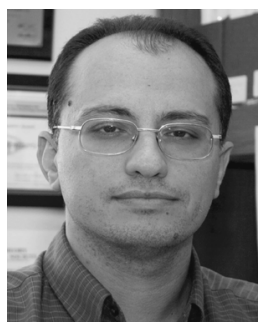

Manos M. Tentzeris (SM'03) received the Diploma degree in electrical and computer engineering (magna cum laude) from the National Technical University of Athens, Athens, Greece, and the M.S. and Ph.D. degrees in electrical engineering and computer science from the University of Michigan, Ann Arbor.

He is currently a Professor with School of Electrical and Computer Engineering, Georgia Institute of Technology (Georgia Tech), Atlanta. He has published more than 320 papers in refereed journals and conference proceedings, four books and 17 book chapters. He has helped develop academic programs in highly integrated/multilayer packaging for RF and wireless applications using ceramic and organic flexible materials, paper-based RFIDs and sensors, Microwave MEMs, SOP-integrated (UWB, mutliband, conformal) antennas and adaptive numerical electromagnetics (FDTD, multiresolution algorithms) and heads the ATHENA research group (20 researchers). He is the Georgia Electronic Design Center Associate Director for RFID/Sensors research, and he has been the Georgia Tech NSF-Packaging Research Center Associate Director for RF Research and the RF Alliance Leader from 2003-2006. He was a Visiting Professor with the Technical University of Munich, Germany, for the summer of 2002, where he introduced a course in the area of high-frequency packaging. He has given more than 50 invited talks in the same area to various universities and companies in Europe, Asia, and America.

Dr. Tentzeris was the recipient/co-recipient of the 2009 E.T.S.Walton Award from the Irish Science Foundation, recipient/corecipient of the 2007 IEEE APS Symposium Best Student Paper Award, the 2007 IEEE IMS Third Best Student Paper Award, the 2007 ISAP 2007 Poster Presentation Award, the 2006 IEEE MTT Outstanding Young Engineer Award, the 2006 Asian-Pacific Microwave Conference Award, the 2004 IEEE TRANSACTIONS ON ADVANCED PACKAGING Commendable Paper Award, the 2003 NASA Godfrey "Art" Anzic Collaborative Distinguished Publication Award, the 2003 IBC International Educator of the Year Award, the 2003 IEEE CPMT Outstanding Young Engineer Award, the 2002 International Conference on Microwave and Millimeter-Wave 
Technology Best Paper Award (Beijing, China), the 2002 Georgia Tech-ECE Outstanding Junior Faculty Award, the 2001 ACES Conference Best Paper Award and the 2000 NSF CAREER Award and the 1997 Best Paper Award of the International Hybrid Microelectronics and Packaging Society. He was the TPC Chair for IEEE IMS 2008 Symposium and the Chair of the 2005 IEEE CEM-TD Workshop and he is the Vice-Chair of the RF Technical Committee (TC16) of the IEEE CPMT Society. He is the founder and chair of the RFID Technical Committee (TC24) of the IEEE MTT Society and the Secretary/Treasurer of the IEEE C-RFID. He has organized various sessions and workshops on RF/Wireless Packaging and Integration, RFIDs, Numerical
Techniques/Wavelets, in IEEE ECTC, IMS, VTC, and APS Symposia in all of which he is a member of the Technical Program Committee in the area of "Components and RF." $\mathrm{He}$ is an Associate Editor of the IEEE TRANSACTIONS ON MicrowaVE THEORY AND TECHNIQUES, IEEE TRANSACTIONS ON ADVANCED PACKAGING, and International Journal on Antennas and Propagation. $\mathrm{He}$ is a member of URSI-Commission D, a member of MTT-15 committee, an Associate Member of EuMA, a Fellow of the Electromagnetic Academy, and a member of the Technical Chamber of Greece. 